\title{
Axial Micro-Strain Sensor Based on Resonance Demodulation Technology Via Dual-Mode CMECF
}

\author{
Xiao LIANG ${ }^{1 *}$, Tigang NING ${ }^{2}$, Jingcong $\mathrm{LI}^{1}$, Yang $\mathrm{LI}^{2}$, and Zhiming $\mathrm{LIU}^{3}$ \\ ${ }^{1}$ College of Science, Minzu University of China, Beijing 100081, China \\ ${ }^{2}$ Institute of Lightwave Technology, Beijing Jiaotong University, Beijing 100044, China \\ ${ }^{3}$ The 41st Research Institute of CETC, Qingdao 266555, China \\ "Corresponding author: Xiao LIANG_E-mail: 1xiao151@gmail.com
}

\begin{abstract}
This paper firstly and experimentally demonstrates an in-fiber axial micro-strain sensing head, combined with a Mach-Zehnder interferometer (MZI) based on the concentric multilayer elliptical-core fiber (CMECF). This MZI with a high extinction ratio (about $15 \mathrm{~dB}$ ) is successfully achieved with a CMECF-single mode fiber-CMECF (CSC) structure. The MZI sensor theory and the resonance demodulation technology are systematically described in this paper. In this CSC structure, two sections of the CMECF have a role as the mode generator and coupler, respectively. $\mathrm{LP}_{01}$ and $\mathrm{LP}_{11}{ }^{\text {even, }}$, which have similar excitation coefficients, are two dominated propagating mode groups supported in the CMECF. On account of the distinct dual-mode property, a good stability of this sensor is realized. The detected resonance in the MZI shifts as the axial micro-strain variated due to the strong interaction between higher order modes. High sensitivity of $\sim 1.78 \mathrm{pm} / \mu \varepsilon$ is experimentally achieved within the range of $0 \mu \varepsilon-1250 \mu \varepsilon$, meanwhile, the intensity fluctuation is below $0.38 \mathrm{~dB}$.
\end{abstract}

Keywords: Few-mode fiber; fiber sensor; MZI; axial micro-strain

Citation: Xiao LIANG, Tigang NING, Jingcong LI, Yang LI, and Zhiming LIU, "Axial Micro-Strain Sensor Based on Resonance Demodulation Technology Via Dual-Mode CMECF," Photonic Sensors, 2019, 9(1): 78-88.

\section{Introduction}

Fiber-optic sensors exhibit several distinctive features such as low cost, compact size, high sensibility, anti-erosion, immunity to electromagnetic interference, and multiplexing capabilities, which draw a great deal of attention to strain measuring under harsh conditions. Compared with electrical ones, the fiber strain sensors can be suitable in more wide areas. Up to now, many novel fiber sensing structures for strain induction have been reported, such as sensors based on fiber Bragg gratings (FBGs) [1-3], fiber long-period gratings
(LPGs) [4, 5], Mach-Zehnder interferometers (MZIs) [5-7], fiber tapers [6, 7], Saganac [8], and Fabry-Perot (FP) $[9,10]$ structures. However, the fabrication of LPGs and FBGs requires phase mask, ultraviolet (UV) laser, and post-annealing, so that the productions are generally complex, low-efficiency, and high-cost. In contrary, in-fiber interferometic sensors show great advantages over the others in term of cost, stability, fabrication difficulty, and sensitivity. Typically, in-fiber sensors based on the multimode-singlemode-multimode (MSM) structure get good results on the convenient manufacture and high sensitivity. This structure

Received: 15 May 2018 / Revised: 2 August 2018

(C) The Author(s) 2018. This article is published with open access at Springerlink.com

DOI: $10.1007 / \mathrm{s} 13320-018-0513-\mathrm{x}$

Article type: Regular 
offers a solution to simplify the production process and lowers the cost of fiber strain sensors. Meanwhile, the coupling/decoupling length loosens up its relationship with the arm length of the MZI, which makes sensors easier to design than the singlemode-multimode-singlemode (SMS) structure.

However, due to many interference modes, the in-fiber sensors based on multimode interference (MMI) are inherently difficult to get stable and clean spectrum, which causes relatively poor resolution and limits their application in the remote operation. In recent years, to overcome these shortages, few-mode fibers (FMFs) have attracted a great deal of interests in MZI-based sensors, attributed to few-mode property [11-14]. Particularly, various modified schemes using the FMF to achieve in-fiber interferometric sensors have been reported, including elliptical-core (e-core) fibers [15], multi-core fibers [16, 17], multi-clad fibers [18, 19], side-hole micro-structure fibers [20], and liquid-core optical fibers [21]. Nevertheless, the most conventional circular-core FMF, in which the cross-sectional intensity distribution of the second-order modes is unstable, used in MMI structures, needs specific modes launched [22, 23]. The e-core FMFs would acquire a well-defined intensity distribution, and only two of the four second-order non-degenerate modes could be transmitted in them. Benefit from the intrinsic features, the stable and smooth interference is simply generated [24]. Therefore, the e-core FMFs could be more applicable to fabricate the MSM type in-fiber interferometric sensors.

In this work, we demonstrate a novel CMECF and an axial micro-strain sensor based on a CMECF-single mode fiber-CMECF (CSC) structure. The particularly designed CMECF could only carry only two-mode groups with comparable excitation coefficients. The CSC structure has clean spectra, which is beneficial to stable sensing operation. Thus, it is convenient to measure the resonance wavelength shift. When the axial micro-strain on the structure is varied, the dip of the transmission spectrum is shifted. The axial micro-strain could be demodulated by monitoring the variation. The detailed analysis of the CSC is characterized systematically by the experiments and discussion of the achieved axial micro-strain sensor.

\section{Design and theoretical principles}

\subsection{Structure of the novel CMECF and sensing head}

As one of the most pivotal components of the sensing head, the few-mode CMECF is made by means of a combination of the conventional modified chemical vapor deposition (MCVD) and solution-doping techniques. Figure 1 shows the microscopic image and measured refractive index difference (RID) distribution of the cleaved cross-section of the CMECF. As depicted in Fig. 1(a), it consists of a conventional circular cladding and an elliptical core area which has a concentric elliptical core and three inner elliptical rings. The diameter of the elliptical core area along the elliptical slow/fast-axes is $22.64 / 18.05 \mu \mathrm{m}$, and the cladding diameter is $125 \mu \mathrm{m}$. The ratio of the fast to the slow axis of this area is 0.797 , and the slow axes of each layer from the inside out are shown below: $a=$ $4.63 \mu \mathrm{m}, b=9.06 \mu \mathrm{m}, c=15.85 \mu \mathrm{m}$, and $d=22.64 \mu \mathrm{m}$. Figure 1(b) shows the measured RID between the conventional cladding and the core area in the slow-axis direction, which is measured by an optical fiber analyzer (EXFO NR9200). The refractive index (RI) of the conventional cladding is 1.444 . The raised index, whose RID is about $4.5 \times 10^{-4}$, could be doped by germanium, and the reduced index, whose RID is about $3 \times 10^{-4}$, could be doped by fluorine. Such an index distribution design, different with step-index fibers, enables not only few-mode feature but also approximately similar mode excitations. 
The particularly designed CMECF could only carry the $\mathrm{LP}_{01}$ and $\mathrm{LP}_{11}{ }^{\text {even }}$ mode groups. According to the actual RID distribution and the fiber structure, the relationship between the effective indices $n_{\text {eff }}$ and wavelengths $\lambda$ of the existing modes is analyzed and calculated by the finite element method (FEM) as shown in Fig. 1(c). As a common sense, guided modes are cut off when the effective index of modes is below cladding one, 1.444. Therefore, as depicted in Fig. 1(c), the higher-order modes, $\mathrm{LP}_{11}{ }^{\text {even }}$ group and $\mathrm{LP}_{11}{ }^{\text {odd }}$ group, have different cutoff wavelengths, and $\mathrm{LP}_{11}{ }^{\text {odd }}$ group is firstly suppressed by elliptical fast-axis [25]. Consequently, the novel CMECF, in which only $\mathrm{LP}_{01}$ and $\mathrm{LP}_{11}{ }^{\text {even }}$ groups shown in the inset could be guided, achieves a distinct dual-mode operation with a range of $1425 \mathrm{~nm}$ to $1575 \mathrm{~nm}$. Attributed to the interference principle, the stable clean interference patterns would be easily got by sensors based on few-mode fibers. On the basis of the foregoing, the distinct dual-mode CMECF is particularly suitable to develop sensor heads based on the MMI.

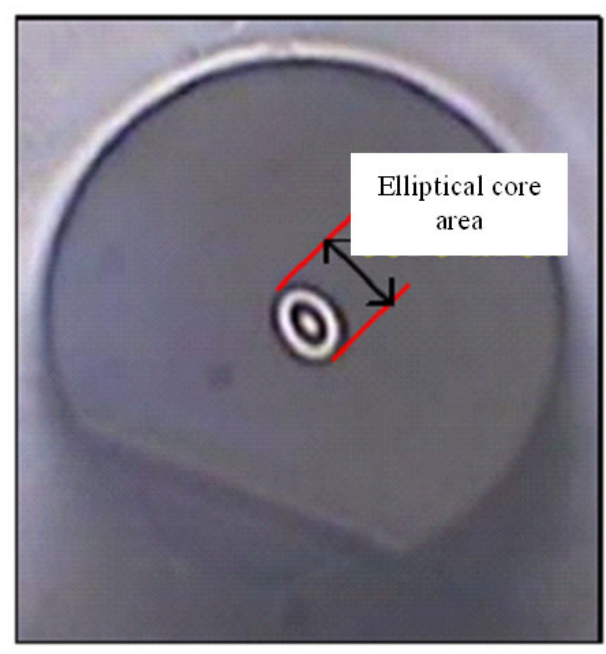

(a)

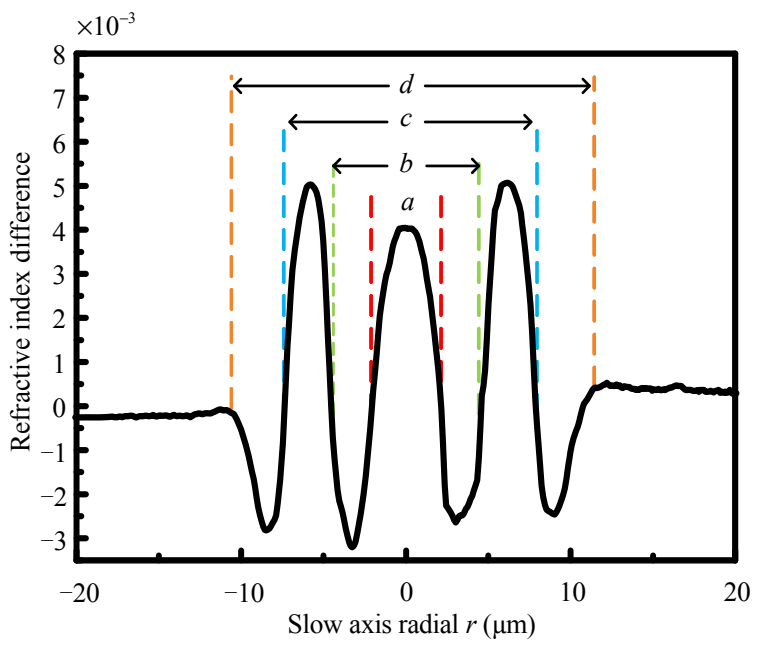

(b)

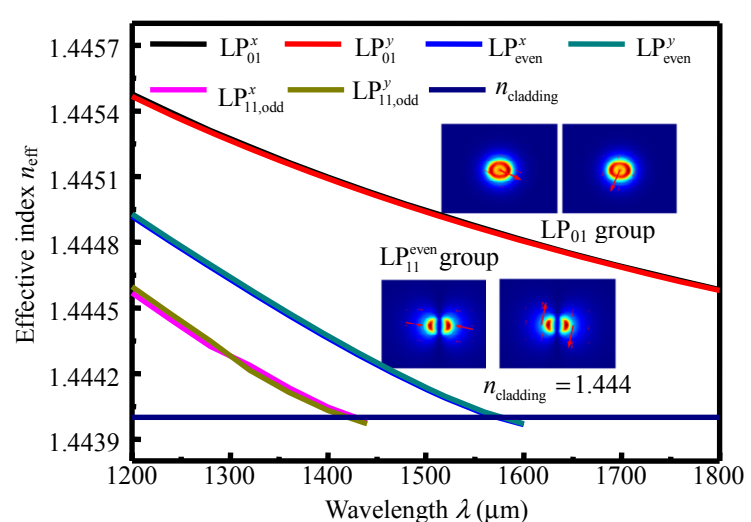

(c)

Fig. 1 Schematics of (a) cross-section view, (b) refractive index distribution of the CMECF, and (c) curves of $\lambda$ vs. $n_{\text {eff }}$ of the guided modes. 
Figure 2 shows the structure schematic of an in-fiber MZI based on CMECFs, which plays a role of the sensing head. It is manufactured by splicing a segment of the SMF (corning SMF-28) between two same length segments of CMECFs using the fiber fusion splicer (Fujikura, FSM-100M/P), and a CSC structure is constructed. As shown in the insert of Fig. 2, the CMECFs act as the mode generator and coupler, and the central SMF acts as the arm of the MZI. An input SMF and an output one are respectively spliced with the ends of the CSC structure. To achieve a perfect interference spectrum and a sensitive response to surrounding variations, coatings attached to the sensing head are entirely removed. The losses introduced by fused splice between the CMECF and SMFs are $0.16 \mathrm{~dB}, 0.56 \mathrm{~dB}$, $0.2 \mathrm{~dB}$, and $0.16 \mathrm{~dB}$, respectively, which correspond to Point 1 to Point 4 as indicated in Fig. 2. The lengths of two CMECFs are both $5 \mathrm{~mm}$, and the central SMF is $38 \mathrm{~mm}$. The overall length is only $48 \mathrm{~mm}$, which could be widely used in a compact sensor system.

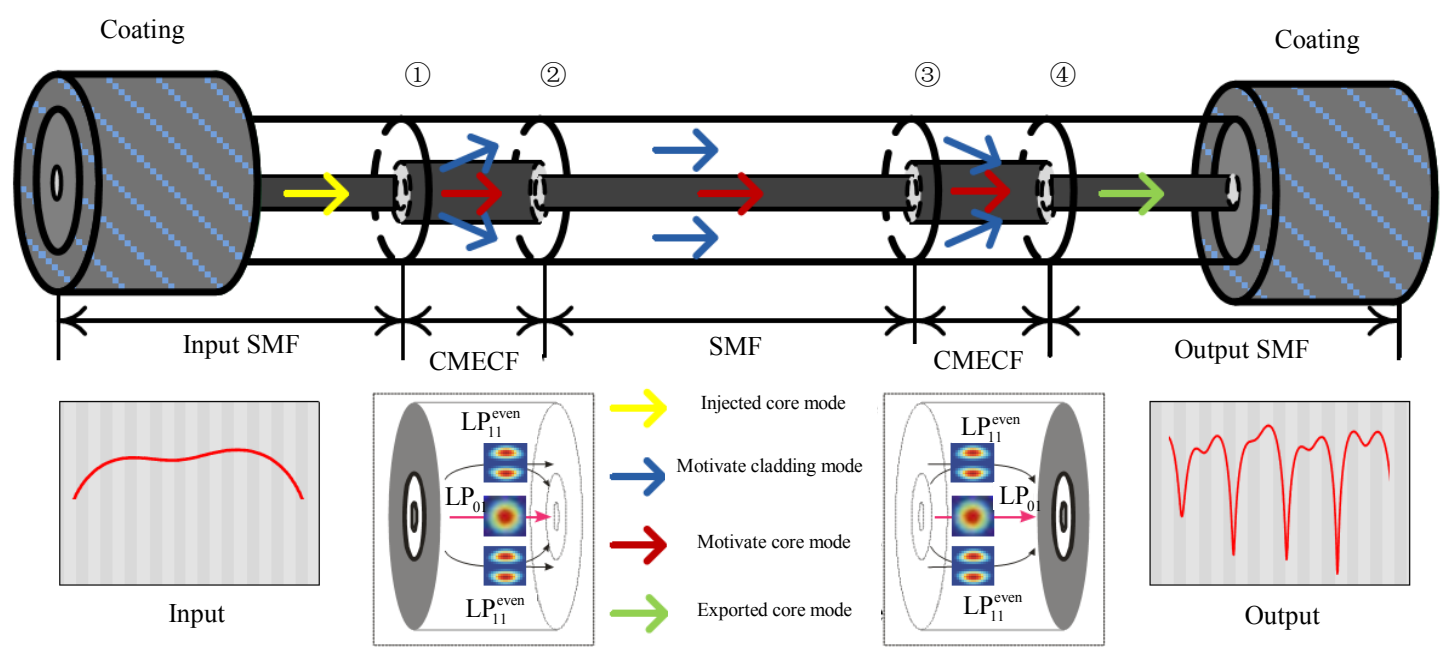

Fig. 2 Structures of MZI (the inset is excited and coupled modes in the CMECF).

Figures 3(a) and 3(b) show the field evolution and the normalized power along the CSC, respectively. Figure 3(c) is a larger version of the SMF-CMECF structure. As depicted in Fig. 1(b), it can be seen that the index distribution of the CMECF is non-axisymmetric. Even if the CMECF is coaxially spliced with an SMF, there is a significant core offset between those two fibers at Point 1 . Therefore, similar to the axially symmetric $\mathrm{LP}_{01}$ mode, the dominated higher-order mode group $\mathrm{LP}_{11}{ }^{\text {even }}$ can be preferentially excited and confined in the elliptical core area, which reveals the interference of the power distribution between two dominant guided modes. And only a few subordinated high-order modes are excited due to the waveguide property. As depicted in Figs. 2 and 3, when the excited modes are coupled into the central SMF at Point 2, $\mathrm{LP}_{01}$ mode is coupled into its core, and the high-order modes are coupled into its cladding. The stable propagation is performed over different optical paths in the central SMF. Then, the vast majority of modes are eventually coupled back into the core of the output SMF through the second section $\mathrm{CMECF}$ at Point 3 and beat with each other. Due to the optical path difference between the $\mathrm{LP}_{01}$ and other high-order modes in the output SMF, a stable interference pattern would be caused. As depicted in Fig. 3(c), the energy is concentrated in the core area, and preliminary estimate reveals that the dominated interference modes are $\mathrm{LP}_{01}$ and $\mathrm{LP}_{11}{ }^{\text {even }}$ whose field distributions are mainly focused in the core area of the CMECF. 


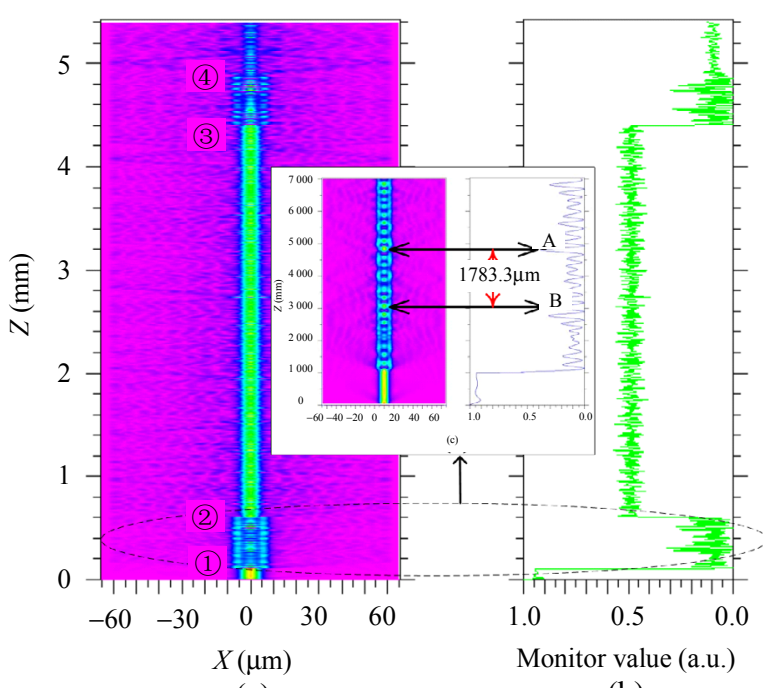

(a)

(b)

Fig. 3 Interference process in this MZI: (a) field evolution, (b) normalized power along the CSC, and (c) a larger version of the SMF-CMECF structure.

According to the property of the CMECF, the incident light excites a complete set of guided high-order modes. The energy proportion of guided modes has magnitudes of difference. The excitation coefficient could be expressed as the energy coupling coefficient determined by two-dimension overlap integral between the incident filed and each guided mode distribution, which is given as [26, 27]

$$
\eta_{n}=\frac{\iint_{S} \psi_{S}(x, y) \psi_{n}(x, y) d x d y}{\iint_{S} \psi_{n}(x, y) \psi_{n}(x, y) d x d y}
$$

where $\psi_{S}(\mathrm{x}, \mathrm{y}), \psi_{n}(\mathrm{x}, \mathrm{y})$, and $\eta_{n}$ are the incident filed distribution, the guided mode distribution, and excitation coefficient of the $n$th order mode in the CMECF, respectively.

Calculated by (1), the excitation coefficients are depicted in Fig.4(a). As we can see from the results, the excitation coefficients of the $\mathrm{LP}_{01}$ and $\mathrm{LP}_{11}$ even are the highest, and the total of these two modes is up to $77 \%$ energy of the input power. That is to say, there are only two dominated interference modes supported by the CMECF. Therefore, the spectrum can be simplified as a paired interference process [28]. The measured transmission spectrum of this MZI in air is shown in Fig. 4(b). According to the definition, the half beat length $L_{\pi}$ would be simply described as [26]

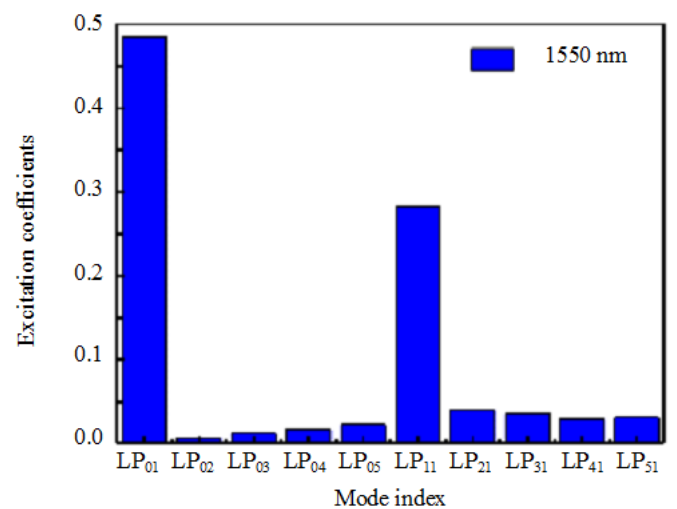

(a)

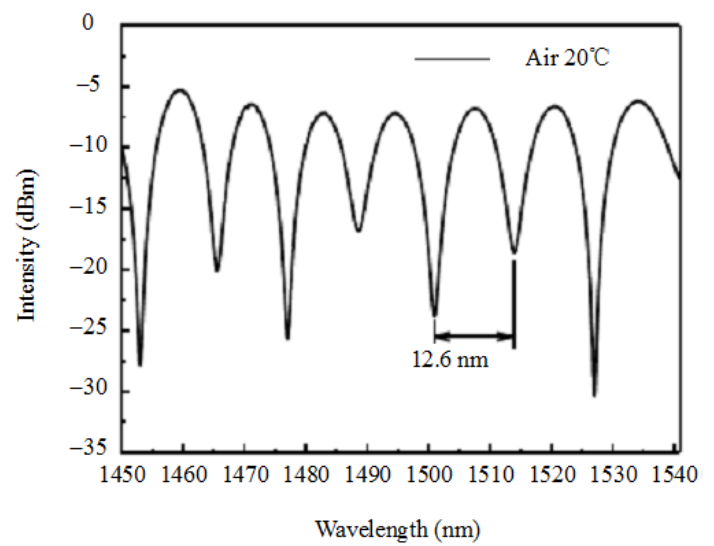

(b)

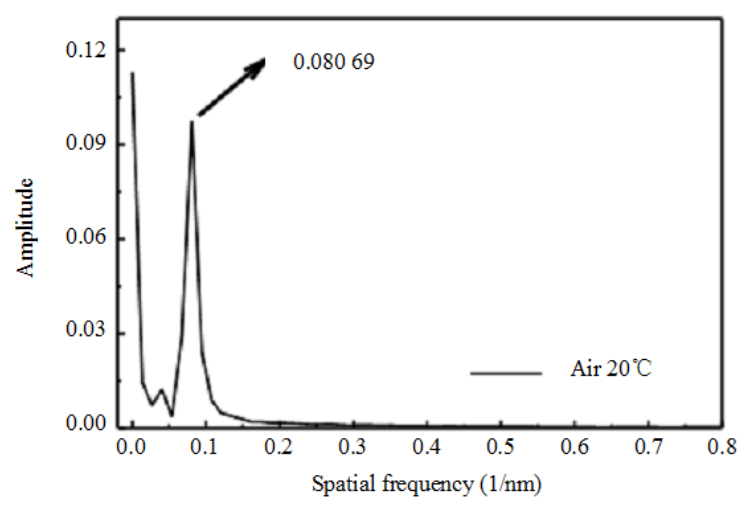

(c)

Fig. 4 Interference-pattern analysis: (a) excitation coefficients of guided modes in the CMECF, (b) transmission spectrum of the CSC structure, and (c) spatial frequency of the transmission spectrum.

$$
L_{\pi}=\frac{\pi}{\beta_{L P_{01}}-\beta_{L P_{11}{ }^{\text {even }}}}
$$

where $\beta_{L P_{01}}$ and $\beta_{L P_{11}{ }^{\text {even }}}$ are propagation constants which can be calculated by the effective indices of $\mathrm{LP}_{01}$ and $\mathrm{LP}_{11}{ }^{\text {even }}$ in the CMECF. As the length is fixed to the odd times of $\frac{1}{2} L_{\pi}$ of the CMECF, the 
coupling ratios would reach $3 \mathrm{~dB}$, so that the contrast of the interference spectrum could attain its maximum performance according to the coupled mode theory [19]. Therefore, using the distinct dual-mode CMECF, the design of the sensing head would be more flexible, and the construction parameters are easier to be controlled than other multimode fibers. As depicted in Fig. 3(c), Points A and $\mathrm{B}$, which are corresponding to adjacent maximum power points of the light field along the $\mathrm{CMECF}$, are the reimaging points of the interference spectra. Obviously, the beat length $L_{2 \pi}$ is equivalent to the distance between two adjacent highest power points, which is approximately $1.7833 \mathrm{~mm}$. As described above, the length of the CMECF, $5 \mathrm{~mm}$, is approximate 11 times of $L_{\pi} / 2$, and the length of the inserted SMF is $38 \mathrm{~mm}$. The total length of this filter is only $48 \mathrm{~mm}$, which is so tiny that it can be applied in a compact sensor system.

In Fig. 4(b), the distance of adjacent resonance dips has a nearly consistent value of $12.6 \mathrm{~nm}$, which is the free spectral range (FSR) related to the length of the SMF in the CSC. As we can see, the stable interference is caused, and the relatively clean transmission spectrum is got. And the approximate gain flattening peaks in intensity are roughly obtained due to the close excitation coefficients of the two-mode groups. It is easily explained by the distinct dual-mode interference process which will cause optical power exchange between the fundamental mode and the high-order modes. Therefore, the FSR would be approximately described as

$$
F S R \approx \frac{\lambda^{2}}{\left(n_{\mathrm{LP}_{01}, \mathrm{SMF}}-n_{\mathrm{LP}_{11}^{\text {even }}, \mathrm{SMF}}\right) L_{\mathrm{SMF}}+2\left(n_{\mathrm{LP}_{01}, \mathrm{EMCF}}-n_{\mathrm{LP}_{11}^{\text {even }}, \mathrm{EMCF}}\right) L_{\mathrm{EMCF}}}
$$

where $n_{\mathrm{LP}_{01}, \mathrm{SMF}}, n_{\mathrm{LP}_{11}^{\text {even }, \mathrm{SMF}}}, n_{\mathrm{LP}_{01}, \mathrm{EMCF}}$, and $n_{\mathrm{LP}_{11}^{\text {even }}, \mathrm{EMCF}}$ are the effective indices of $\mathrm{LP}_{01}$ and $\mathrm{LP}_{11}{ }^{\text {even }}$ in the SMF and CMECF of the CSC structure, respectively. From (3), we can see that the FSR is easy to be adjusted by the length of the SMF/CMECF. Analyzed by finite-different time-domain (FDTD), the transmission spectrum of the CSC structure using different lengths of the SMF is calculated, which is depicted in Fig. 5. It could be found out from the simulation result that the FSR is inversely proportional to the length of the SMF. And the implementation of effective control of the FSR can easily be got by changing the length of the SMF. The method is simple, rapid, sensitive, and accurate for optimizing experimental condition.

In order to further verify the distinct dual-mode property, Fig.4(c) shows that the spatial frequency is

$$
I=I_{\text {fundamental }}+\sum_{m} I_{\text {high-order }}^{m}+\sum_{m} 2 \sqrt{I_{\text {fundamental }} I_{\text {high-order }}^{m}} \cos \phi^{m}
$$

where $I_{\text {fundamental }}$ and $I_{\text {high-order }}^{m}$ are the intensities of fundamental and the $m$ th high-order modes, respectively. $\phi^{m}$ is the phase delay of any two obtained by transforming the spectrum with fast Fourier transformation (FFT). Obviously, there are only two peaks corresponding to $\mathrm{LP}_{01}$ and $\mathrm{LP}_{11}{ }^{\text {even, }}$, respectively. The distance between the two peaks is $0.08069 / \mathrm{nm}$, the reciprocal of which equals to the FSR of the MZI. Except the dominated peaks, there are other frequency peaks with relatively low intensities which demonstrate weak mode interferences between the fundamental modes and high-order modes. It is demonstrated experimentally that only two major modes, $\mathrm{LP}_{01}$ and $\mathrm{LP}_{11}{ }^{\text {even }}$, are supported by the CMECF.

\subsection{Sensing principle}

The basis of the sensing principle is the concept of MMI effect in the CSC structure. According to the interference theory, the intensity of the transmission spectrum can be expressed as

modes after transmission along the CSC structure. As the central-SMF length $L_{\mathrm{SMF}}$ is much longer than CMECF's $L_{\mathrm{EMCF}}, \phi^{m}$ could be expressed as [29] 


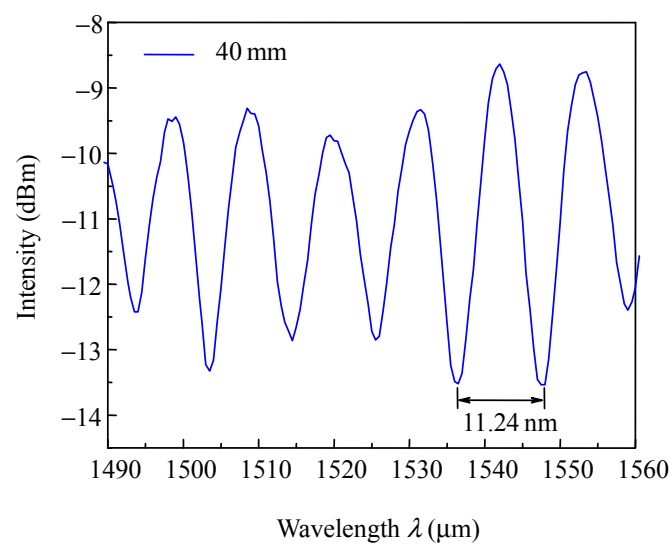

(a)

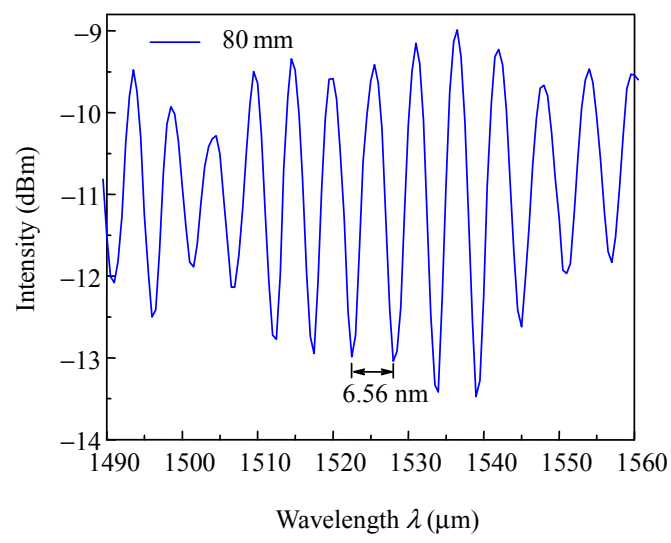

(a)

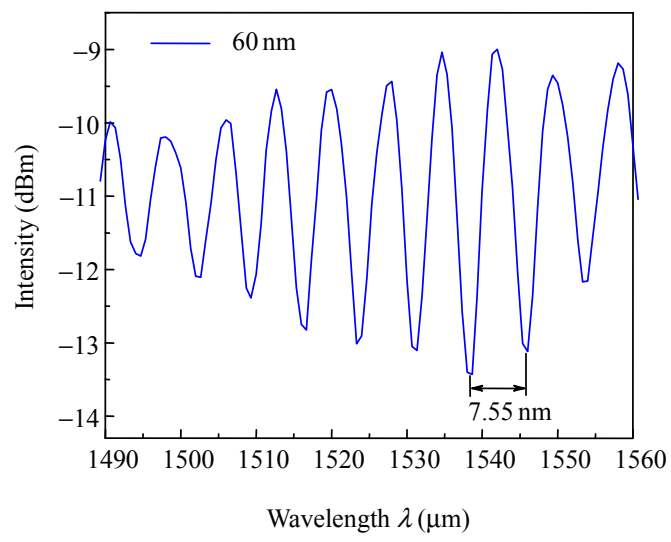

(b)

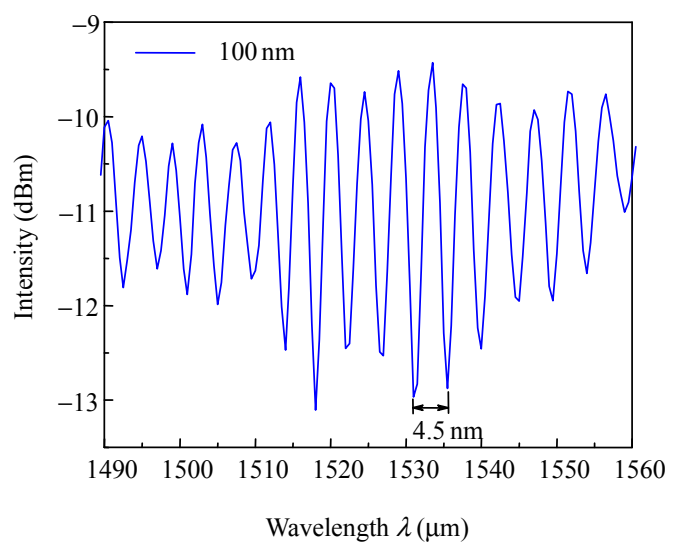

(d)

Fig. 5 Simulations of FSR for different lengths of the SMF in the MSM structure: (a) $40 \mathrm{~nm}$, (b) $60 \mathrm{~nm}$, (c) $80 \mathrm{~nm}$, and (d) $100 \mathrm{~nm}$.

$\phi^{m}=\frac{2 \pi\left(n_{\mathrm{eff}}^{\text {fundamental }}-n_{\mathrm{eff}}^{\text {high-order,m }}\right) L_{\mathrm{SMF}}}{\lambda_{m}}=\frac{2 \pi \Delta n_{\mathrm{eff}}^{m} L_{\mathrm{SMF}}}{\lambda_{m}}$

where $\lambda_{m}$ is the operation wavelength, $L_{\mathrm{SMF}}$ is the length of the SMF, and $\Delta n_{\text {eff }}^{m}$ is the effective index difference between the fundamental and $m$ th high-order modes. As $\phi^{m}=2 q \pi$, the output spectrum would reach its maximum, and the wavelength of the $q$ th order peak could be expressed as follows [29]:

$$
\lambda_{m}=\frac{\Delta n_{\mathrm{eff}}^{m} L_{\mathrm{SMF}}}{q} .
$$

Equation (6) shows that the wavelength is related with the fiber length and the refractive index. With the axial-strain applied on fibers, the length and index of the CSC structure could be changed. Therefore, the relative wavelength could be changed by weak disturbance of strain in ambient using the proposed model. The axial-strain measurement would be achieved by the in-fiber sensing head. To exclude the negative influences on surrounding temperature and refractive index changes, the wavelength variation caused by axial micro-strain can be obtained, which can be expressed as [30]

$$
\frac{\Delta \lambda_{m}}{\lambda_{m}}=-\left(1+2 \mu+p_{\mathrm{e}}\right) \varepsilon
$$

where $\varepsilon=\frac{\Delta L_{\mathrm{SMF}}}{L_{\mathrm{SMF}}}$ is the axial micro-strain, $\mu$ is the Poisson ratio, and $p_{e}$ is the effective strainoptic coefficient. For silica fibers, the parameters $\mu=0.16$ and $p_{e}=0.22$ are generally valued. Thus, the length change caused by the axial micro-strain makes the resonance dip wavelength shift and resonance dip amplitude unchangeableness. In the other words, the axial micro-strain can be 
demodulated by monitoring the variation of the wavelength at which the minimum interference occurs. As depicted in Fig.6, the simulation results on axial micro-strain sensing are given by the FDTD. In the CSC structure, the lengths of two CMECFs are both $5 \mathrm{~mm}$, and the central SMF is $50 \mathrm{~mm}$. This CSC structure is elongated at a step of $20 \mu \mathrm{m}$, corresponding to the axial micro-strain of $400 \mu \varepsilon$.

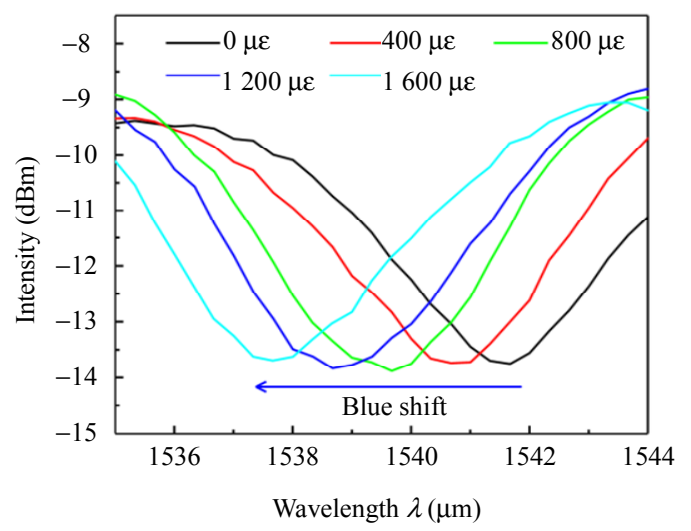

(a)

Fig. 6 Simulations of axial micro-strain sensing: (a) wavelength blue shift and (b) the curve of the micro-strain vs. wavelength.

\section{Experimental methods and measurement}

\subsection{Experimental methods}

Figure 7 shows the experimental architecture of the axial micro-strain measurement at the room temperature. The sensing head based on the in-fiber MZI, fabricated in Section 2.1, is used in this experiment, in which the lengths of two CMECFs are both $5 \mathrm{~mm}$, and the central SMF is $38 \mathrm{~mm}$. The sensing head is fastened by two fiber clamps, and one end of the structure can be elongated by the micro-adjuster. This initial state is used to calibrate the strain, where the axial micro-strain exerted on

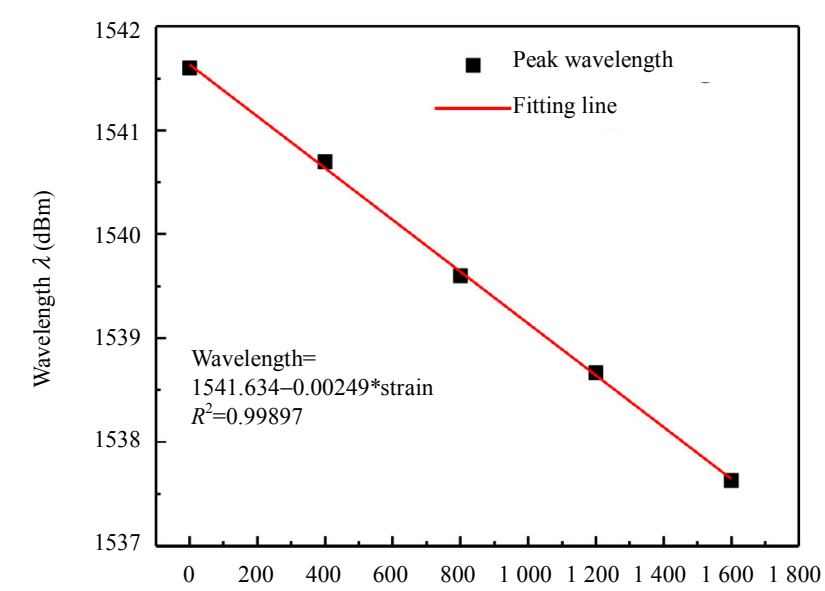

(b)
Figure 6(a) shows the wavelength blue shift by the axial micro-strain increasing, and the strain is linearly related to the wavelength drift as shown in Fig. 6(b), which is in accord with the theoretical analysis ones. It is a liner function relationship with the sensitivity of about $2.49 \mathrm{pm} / \mu \varepsilon$. Based on this feature, the CSC structure can be applied for axial strain sensing.

the sensing head is $0 \mu \varepsilon$. In the linear configuration, a broadband light source (KOHERAS, superK uersa) is used as the injection light, and a spectrometer (YOKOGAWA, AQ6375) is used to detect the transmission spectrum. The distance between two fiber clamps is $9.6 \mathrm{~cm}$. For uniform strain, each micro-adjuster elongates the CSC structure at a step of $10 \mu \mathrm{m}$, and the total elongating length is $20 \mu \mathrm{m}$, corresponding to axial micro-strain of $208 \mu \varepsilon$. By monitoring the dips variation utilizing the optical spectrum analyzer (OSA), the ambient perturbation of axial micro-strain could be obtained distinctly.

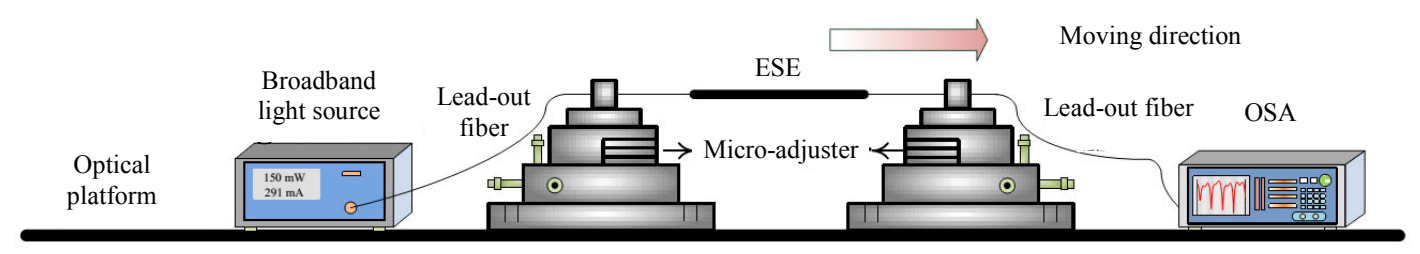

Fig. 7 Configuration of the proposed CSC structure based sensing system. 


\subsection{Measurements and results}

The experiment is performed at the room temperature with a good vibration isolation. Figure 8(a) shows that, as the axial micro-strain is increased from $0 \mu \varepsilon$ to $1250 \mu \varepsilon$ at an interval of $208 \mu \varepsilon$, the wavelength shift is simultaneously recorded from $1539.3 \mathrm{~nm}$ to $1541.5 \mathrm{~nm}$. To avoid the measuring error produced by the strain fluctuation, each measurement is held for a period of time. Caused by the stretching of the sensing head, the length change becomes larger. According to (7), the interference wavelength would be led to blue shift as the axial strain increases. Meanwhile, the amplitudes of resonance dips are flat and achieve a high extinction ratio $(\sim 15 \mathrm{~dB})$. The sensor is configured for C-band communications, which has potential applications in the remote sensing system.

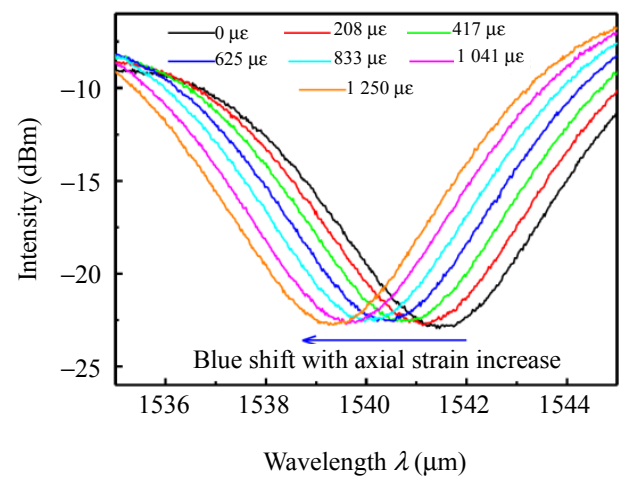

(a)

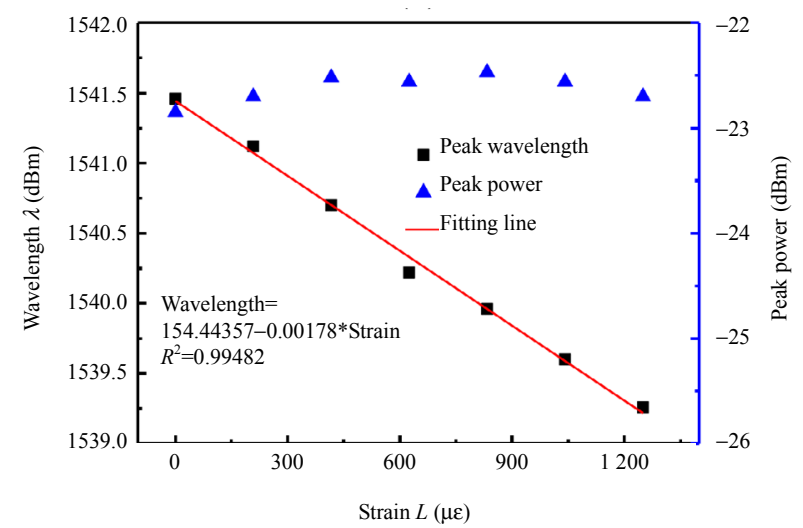

(b)

Fig. 8 Experimental results: (a) wavelength blue shift depending on micro-strain increase and (b) the curve of the micro-strain vs. wavelength (solid line) and variation of peak power during the measurement (dotted lines).
As depicted in Fig. 8(b), the relationship is obtained between the axial strain and the wavelength using a line fitted by solid squares. It is a liner function relationship with the sensitivity of about $1.78 \mathrm{pm} / \mu \varepsilon$, which is less than the one in simulation in Fig. 6(b). The major reason of the deviation is that the stretch region in simulation is not inconsistent with the one in the experiment. Meanwhile, there would be inherent errors in simulation/experiment. In addition, there are some derivations from the linearity. One of the reasons is that the axial strain exerted on the sensing head is not uniformity induced from the imperfect of the fiber clamps. The correlation coefficient $\left(R^{2}=0.99482\right)$ of polynomial fitting is so close to one that the linearity of this axial-strain sensing system is very accurate. The mechanism of the axial micro-strain sensing is mainly based on the distinct dual-mode interference, and thus the dip gain has a fine adjustment. The fluctuation of dip gains is below $0.38 \mathrm{~dB}$ as depicted in Fig. 8(b) (dotted triangles) by the end of the experiment.

\subsection{Discussion}

In general, available in-fiber sensors based on the MMI are difficult to get stable and clean spectrum, but available with highly sophisticated structure, such as fiber tapers and core-offset splices $[31,32]$. In this paper, a simple and good stable sensor for axial micro-strain detection based on the resonance demodulation technology is successfully achieved. Differing from the structure via conventional fibers commonly known as small size and low cost, this CSC structure has advantages of higher extinction ratio, and high strain sensitivity and replicability. In simulations and experiments, the approximate gain flattening peaks of the CSC are obtained easily. A detailed investigation into the distinct dual-mode interference process in the CSC is therefore necessary. Also, all the measurements reported here are performed in single-variable cases, and the effect of the presence in multi-variable cases 
is ignored.

\section{Conclusions}

An axial micro-strain sensing head combined with a tiny in-fiber MZI has been demonstrated. This in-fiber MZI is made of the distinct dual-mode CMECFs. The designed index distribution of the CMECF supports only the fundamental mode and $\mathrm{LP}_{11}{ }^{\text {even }}$ mode, and ensures the similar excitation coefficients of these two modes, leading to stable transmission. Based on these characteristics, the stable interference is produced so as to get the cleaner spectrum, and a high extinction ratio $(\sim 15 \mathrm{~dB})$ is obtained. Based on the resonance demodulation technology, the experiments on sensing axial micro-strain using this tiny MZI based on the CSC structure are illustrated. The axial micro-strain has a linear relationship with the resonance dips, and the sensor has an experimental sensitivity of $\sim 1.78 \mathrm{pm} / \mu \varepsilon$ in the range from $0 \mu \varepsilon$ to $1250 \mu \varepsilon$. Not only are the shifted dips in the C-band range, but also they have the additional advantages of slight fluctuations (below $0.38 \mathrm{~dB}$ ) in intensity. Therefore, the proposed sensing system possesses potential applications in the remote operation sensing system.

\section{Acknowledgment}

This work was supported by the Major State Basic Research Development Program of China (Granted No. 2010CB328206), the "Double Tops" Construction Funds of Minzu University of China (Granted No. 018004032101), the Young Teachers' Research Project of Minzu University of China, and in part by the Fundamental Research Funds for the Central Universities, China.

Open Access This article is distributed under the terms of the Creative Commons Attribution 4.0 International License (http://creativecommons.org/licenses/by/4.0/), which permits unrestricted use, distribution, and reproduction in any medium, provided you give appropriate credit to the original author(s) and the source, provide a link to the Creative Commons license, and indicate if changes were made.

\section{References}

[1] L. Rindorf and O. Bang, "Sensitivity of photonic crystal fiber grating sensors: biosensing, refractive index, strain, and temperature sensing," Journal of the Optical Society of America B, 2008, 25(3): 310-324.

[2] D. P. Zhou, J. W. Y. Lit, L. Wei, W. K. Liu, and Y. Liu, "Simultaneous measurement for strain and temperature using fiber Bragg gratings and multimode fibers," Applied Optics, 2008, 47(10): 1668-1672.

[3] G. L. Yin, J. Tang, C. R. Liao, G. J. Wang, and Y. P. Wang, "Determination of optical fiber parameters based on fiber gratings and a search procedure," Journal of Lightwave Technology, 2017, 35(16): 3591-3596.

[4] Y. P. Wang, L. Xiao, D. N. Wang, and W. Jin, "Highly sensitive long-period fiber-grating strain sensor with low temperature sensitivity," Optics Letters, 2006, 31(23): 3414-3416.

[5] G. S. Barabach, D. Kowal, M. K. Szczurowski, P. Mergo, and W. Urbanczyk, "Hydrostatic pressure and strain sensitivity of long period grating fabricated in polymer microstructured fiber," IEEE Photonics Technology Letters, 2013: 25(5): 496-499.

[6] W. Cui, J. H. Si, T. Chen, and X. Hou, "Compact bending sensor based on a fiber Bragg grating in an abrupt biconical taper," Optics Express, 2015, 23(9): 11031-11036.

[7] G. L. Yin, S. Q. Lou, and H. Zou, "Refractive index sensor with asymmetrical fiber Mach-Zehnder interferometer based on concatenating single-mode abrupt taper and core-offset section," Optics \& Laser Technology, 2013, 45: 294-300.

[8] X. Y. Dong, H. Y. Tam, and P. Shum, "Temperature-insensitive strain sensor with polarization-maintaining photonic crystal fiber based Sagnac interferometer," Applied Physics Letters, 2007, 90(15): 151113-1-151113-3.

[9] M. Deng, C. P. Tang, T. Zhu, and Y. J. Rao, "PCF-based Fabry-Pérot interferometric sensor for strain measurement at high temperatures," IEEE Photonics Technology Letters, 2011, 23(11): 700-702.

[10] T. Zhao, Y. Gong, Y. J. Rao, Y. Wu, Z. L. Ran, and H. J. Wu, "Fiber-optic Fabry-Perot strain sensor based on graded-index multimode fiber," Chinese Optics Letters, 2011, 9(5): 11-14.

[11] X. Liang, S. Liu, Y. Li, Z. B. Liu, and S. S. Jian, "Characteristics of a high extinction ratio comb-filter based on $\mathrm{LP}_{01}-\mathrm{LP}_{11}$ even mode elliptical multilayer-core fibers," Optical Fiber Technology, 2015, 21: 103-109.

[12] P. Hlubina and P. Prochazka, "Sensor applications of two-mode fiber in the Michelson interferometer configuration," SPIE, 1994, 2341: 202-211. 
[13] P. Guo, Y. J. Rao, D. Y. Li, Y. Gong, G. D. Peng, Y. Wu, et al., "Inscription of Bragg gratings in few-mode optical fibers," Chinese Optics Letters, 2013, 11(2): 020606-1-020606-3.

[14] G. L. Yin, C. L. Wang, Y. H. Zhao, B. Q. Jiang, T. Zhu, Y. P. Wang, et al., "Multi-channel mode converter based on a modal interferometer in a two-mode fiber," Optics Letters, 2017, 42(19): 3757-3760.

[15] D. S. Moon, B. K. Kim, A. Lin, G. Sun, W. T. Han, Y. G. Han, et al., "Tunable multi-wavelength SOA fiber laser based on a Sagnac loop mirror using an elliptical core side-hole fiber," Optics Express, 2007, 15(13): 8371-8376.

[16] K. Takenaga, Y. Sasaki, N. Guan, S. Matsuo, M. Kasahara, K. Saitoh, et al., "Large effective-area few-mode multicore fiber," IEEE Photonics Technology Letters, 2012, 24(21): 1941-1944.

[17] C. Xia, R. A. Correa, N. Bai, E. A. Lopez, D. M. Arrioja, A. Schulzgen, et al., "Low-crosstalk few-mode multi-core fiber for high-mode-density space-division multiplexing," in Proceeding of 2012 38th European Conference and Exhibition on Optical Communications, Amsterdam, Netherlands, 2012, pp. 1-3.

[18] C. F. Wei, G. B. Lin, X. P. Dong, and S. C. Tao, “A tunable polarization-independent comb filter based on high-order mode fiber," Journal of Optics, 2013, 15(5): 055403-1-055403-6.

[19] Q. W. Zhang, X. L. Zeng, F. F. Pang, M. Wang, and T. Y. Wang, "Switchable multiwavelength fiber laser by using a compact in-fiber Mach-Zehnder interferometer," Journal of Optics, 2012, 14(4): 045403-1-045403-5.

[20] P. Hlubina, T. Martynkien, J. Olszewski, P. Mergo, M. Makara, K. Poturaj, et al., "Spectral-domain measurements of birefringence and sensing characteristics of a side-hole microstructured fiber," Sensors, 2013, 13(9): 11424-11438.

[21] I. Martincek, D. Pudis, D. Kacik, and K. Schster, "Investigation of intermodal interference of LP 01 and LP 11 modes in the liquid-core optical fiber for temperature measurements," Optik - International Journal for Light and Electron Optics, 2011, 122(8): 707-710.

[22] K. A. Murphy, M. S. Miller, A. M. Vengsarkar, and
R. O. Claus, "Elliptical-core two mode optical-fiber sensor implementation methods," Journal of Lightwave Technology, 1990, 8(11): 1688-1696.

[23] G. L. Yin, C. L. Wang, Y. H. Zhao, B. Q. Jiang, T. Zhu, Y. P. Wang, et al., "Multi-channel mode converter based on a modal interferometer in a two-mode fiber," Optics Letters, 2017, 42(19): 3757-3760.

[24] Y. H. Kim and K. Y. Song, "Mapping of intermodal beat length distribution in an elliptical-core two-mode fiber based on Brillouin dynamic grating," Optics Express, 2014, 22(14): 17292-17302.

[25] T. Geisler, M. E. V. Pedersen, and S. Herstrøm, "Measurement of spatial and polarization birefringence in two-mode elliptical core fibers," in Proceeding of 2013 Optical Fiber Communication Conference and Exposition and the National Fiber Optic Engineers Conference, Anaheim, CA, USA, 2013, pp. 1-3.

[26] W. S. Mohammed, A. Mehta, and E. G. Johnson, "Wavelength tunable fiber lens based on multimode interference," Journal of Lightwave Technology, 2004, 22(2): 469-477.

[27] H. Li, G. Ren, S. Atakaramians, B. T. Kuhlmey, and S. Jian, "Linearly polarized single TM mode terahertz waveguide," Optics Letters, 2016, 41(17): 4004-4007.

[28] L. B. Soldano and E. C. Pennings, "Optical multi-mode interference devices based on self-imaging: principles and applications," Journal of Lightwave Technology, 1995, 13(4): 615-627.

[29] C. C. Wong, M. C. Su, and N. S. Lin, "A compact all fiber refractive index sensor based on modal interference," IEEE Sensors, 2012, 3(11): 1-4.

[30] E. Li, "Temperature compensation of multimodeinterference-based fiber devices," Optics Letters, 2007, 32(14): 2064-2066.

[31] D. G. Mina, J. W. Haus, A. Chong, A. Khanolkar, A. Sarangan, and K. Hansen, "Bi-tapered fiber sensor using visible to near infrared light," Sensors and Actuators A: Physical, 2017, 263: 285-290.

[32] J. Liu, D. N. Wang, and Y. Liu, "Sensitivity improvement by fusion splicing of single mode fibers with core offset," Optical Materials Express, 2017, 7(10): 3722-3730. 